\title{
A Requirements Engineering-based Approach for Defining a System Modification Process during Aircraft Operation
}

\author{
Washington Carvalho Tricote Resende, Luís Gonzaga Trabasso, and Marina Mendonça Natalino Zenun \\ Department of Mechanical Engineering, Instituto Tecnológico de Aeronáutica (ITA), São José dos Campos, São \\ Paulo/Brazil \\ E-mail: washingtontricote@gmail.com,gonzaga@ita.br, marina.natalino@hotmail.com
}

\begin{abstract}
From the systems engineering standpoint, an aircraft may be considered a complex system consisting of multiple subsystems operating in an integrated manner, within several operational environments. During the operation, deviations from the design may arise due to the interaction among the aircraft subsystems and their environments. Such deviations require the aircraft manufacturer to redesign a subsystem in order to obtain a solution of high effectiveness and avoid additional costs of a second rework. This paper presents a requirements engineering-based approach to define or modify a process in which an aircraft subsystem is redesigned. The approach is comprised of seven steps, which include the stakeholder, functional and physical analyses previously presented in the total view framework. The proposed activities also include defining the measures of effectiveness (MoEs), as well as a validation and verification (V\&V) strategy for both the system and the requirements. The present work highlights the importance of requirements definition and management during a modification that occurs at an advanced life cycle stage. The steps presented may be seen as guidelines either to modify or define a process. Therefore, the way each step is deployed depends upon the complexity of the system and organizational issues. A case study has been carried out to evidence the performance difference between a system modification process currently used at an aerospace company and the process modified with the approach presented herein. The outcome shows that the new process would potentially reduce the previously found problems by around $60 \%$, and it is believed that this figure would be even greater if the process were actually implemented.
\end{abstract}

Keywords: systems modification, requirements engineering, complex systems

\section{Introduction}

A system can be defined as an integrated set of elements, subsystems, or assemblies that accomplish a defined objective. The term elements refers to hardware, software, firmware, processes, people, information, techniques, facilities, services, and other support elements. Systems are organized into a hierarchy, consisting of systems elements, which can be broken down into new system elements until an atomic level [1]. A system is considered complex when the interaction between its components can give rise to large events that cannot be located back in the properties or microlevel behaviour of the components. These emergent effects are really difficult to foresee [2]. An aircraft fits the definition of a complex system and, in this paper, it was considered the higher-level system.

Even though the aircraft life cycle presents its stages in a chronological sequence, as shown in fig. 1, several operational environments and systems interactions may lead to the necessity of modifying some subsystems at an advanced life cycle stage. Thus, despite all development effort, the redesign of aircraft subsystems may be needed during aircraft operation.

Modifying a system at an advanced life cycle stage implies a higher financial impact [1]. Therefore, it is extremely important to have a process that enables the development of successful solutions, avoiding additional costs of a second rework. Besides the financial aspect, a possible second rework could also adversely affect customer satisfaction and, consequently, the corporate image.

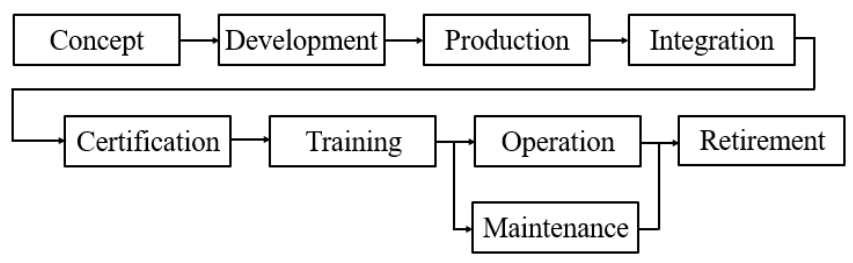

Figure 1: Aircraft life cycle stages. Adapted from [3].

When the operator, e.g. airlines for commercial aviation, reports a system failure to the aircraft manufacturer, the company needs to internalize the problem by involving a 
multidisciplinary team to support the customer and to design a solution of high effectiveness. Although the way in which the issue is internalized depends on the organizational structure of the company, the engineers are the process stakeholders responsible for the system redesign. Thus, the activities to be performed by the engineers shall be well structured and focus on problem solving. This paper uses the requirements engineering, which is a subset of systems engineering, to define a system modification process to be applied during aircraft operation.

\section{Requirements engineering-based approach}

The requirements engineering-based approach presented herein makes use of two frameworks: the total view framework and the framework for a sufficient set of requirements (SCoRe) [3] [4]. The former was built by combining the concepts of both systems and concurrent engineering. The latter is based on the former, but it emphasizes the definition of a sufficient set of requirements. Instead of covering the whole life cycle of the system, the scope of this work focus on an advanced life cycle stage. Thus, the three-dimensional analysis presented in the total view framework was tailored to fit the modification of a system at the operation stage. The stakeholder, functional and physical analysis, i.e. the three-dimensional analysis, is performed focusing on the problems reported by the customers in order to obtain a sufficient set of requirements. Some authors defended that the set of requirements must be complete [5]. As it may be difficult to identify completeness, the term sufficient was presented in the framework SCoRe and it is used here.

The system modification process proposed is shown in fig. 2 . Each step contains several activities that guide the development of a solution of high effectiveness. The first five steps are adapted from the total view framework. The process also includes activities involving the organization of the requirements into a hierarchy and the definition of a verification and validation strategy for both the system and the requirements.

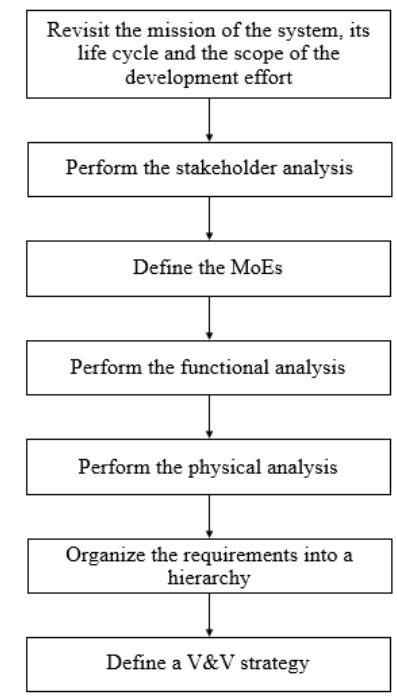

Figure 2: The system modification process
The process presented in fig. 2 can be applied to modify systems of different levels of complexity. When the aircraft is analysed as the higher-level system, it can be broken down into flight controls, avionics, propulsion, environmental control, interior systems, among others. These systems are aircraft subsystems and all of them are subjected to modifications in an advanced life cycle stage. The complexity of the modification will depend on the complexity of the system, thus the seven steps of the system modification process must be adapted according to this complexity. It is equally important to adapt the process taking into account the stakeholders' background and/or company standards. Each step of the system modification process is detailed hereafter.

\subsection{First step of the system modification process}

This step consists of revisiting the mission of the system, its life cycle and the scope of the development effort. The mission of the system states its purpose or reason of being and guides the integrated process [6]. Although the mission of the system is often defined at the beginning of the life cycle, the process proposes revisiting the mission during a system redesign so that the requirements that guide the modification of the system are aligned with the mission. The scope of the development effort evidences other life cycle stages in which the development organization has partial or total responsibility [6].

In this step, the abstraction level of system hierarchy is defined and all subsequent analyses shall be performed for all layers of the hierarchy. When defining the mission and life cycle of an aircraft subsystem, they are within the mission and life cycle of higher-level systems. Therefore, it may be more interesting to define these concepts for the higher-level system.

Even though this paper focus on the operational stage of the life cycle, this is not the only stage to be considered during analysis. Some problems that occur in operation can be related to production issues and may also occur during the maintenance of the system. Hence, it is important to define the stages to be analysed, as well as their different scenarios, which are either the decomposition of a stage or the alternatives in each stage - e.g. preventive and corrective maintenance [6]. The activities within the first step are presented in fig. 3 .
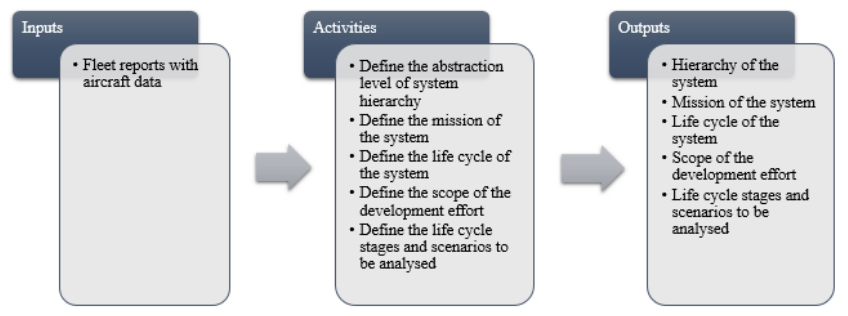

Figure 3: Inputs, activities and outputs of the first step

\subsection{Second step of the system modification process}

In this step, the stakeholder analysis is performed, in which the system and organization stakeholders are identified for each level of system hierarchy and for each stage of the life 
cycle. Stakeholder concerns are used to define the stakeholder requirements [6]. All requirements must be written following a standard so that they are easily and equally understood by all process stakeholders. One way of standardising the language used for requirements is using boilerplates throughout the process [7]. All activities contained in the second step are shown in fig. 4.

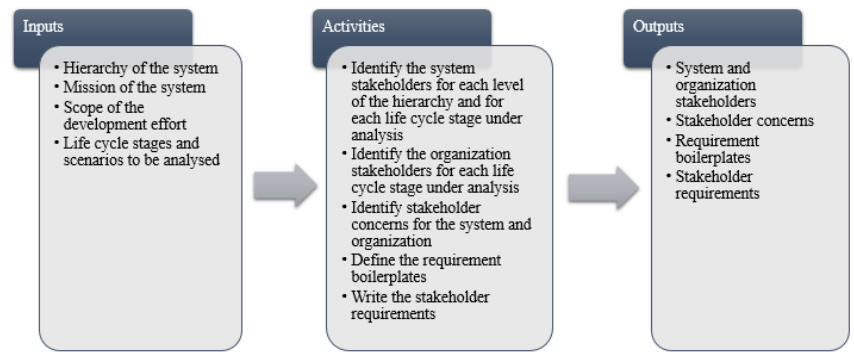

Figure 4: Inputs, activities and outputs of the second step

The definition of stakeholder requirements is within the problem domain, in which any reference to a particular solution is avoided in order to allow the engineers to devise the best solution without preconceived ideas. Functional and physical characteristics of the system are defined afterwards in the solution domain and are stated through system requirements and architectural design [7].

\subsection{Third step of the system modification process}

From the system and organization stakeholders and their concerns, the MoEs and their acceptance criteria are defined, as shown in fig. 5. The MoEs measure how the system meets the stakeholder requirements [6].

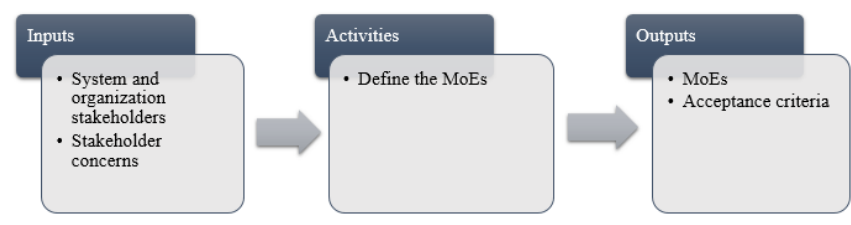

Figure 5: Inputs, activities and outputs of the third step

\subsection{Fourth step of the system modification process}

The functional analysis is within the solution domain and it is performed in this step. The environment elements that interact with the system are identified, as well as information (INF), material (MAT), and energy (ENR) exchanged between these elements and the system. The functional context diagrams are made for the system and organizations for each life cycle stage under analysis. For the system, each hierarchy level must be considered [6].

To completely represent the system functioning, the systems engineers' activities also include doing the list of events, the functional structure diagram, and the state transition diagram. This way, at the end of this step, the engineers will be able to know the environment elements that interacts with the system, the way in which the interactions occur, as well as the functions and states of the system. With all this information, the first subset of system requirements is written. In this step, the interface requirements among the system and environment elements are captured. All activities contained in this step are listed in fig. 6.
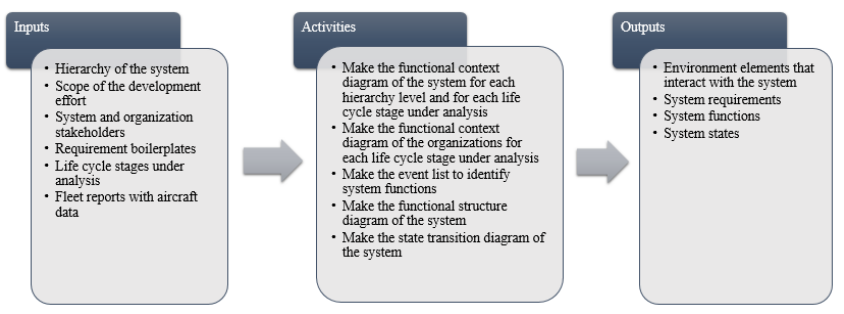

Figure 6: Inputs, activities and outputs of the fourth step

\subsection{Fifth step of the system modification process}

The physical analysis consists of making the architecture context diagram by identifying the physical components responsible for the exchange of INF, MAT and ENR between the system and the environment elements [6]. The second subset of system requirements is defined. The activities to be performed in this step are presented in fig. 7.

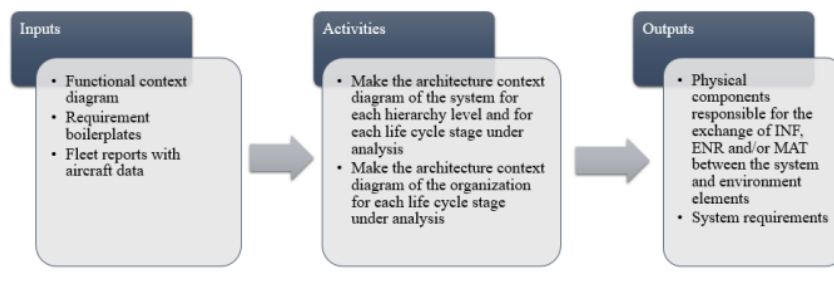

Figure 7: Inputs, activities and outputs of the fifth step

\subsection{Sixth step of the system modification process}

At this point, all stakeholders and systems requirements will have been defined. Thus, it is necessary to organize all these requirements into a unique hierarchy, allowing correct requirements traceability, as shown in fig. 8. Stakeholder requirements are at the highest level and there is no hierarchical difference among them. On the other hand, system requirements are organized according to the system hierarchy. The highest level system requirements are linked to stakeholder requirements.

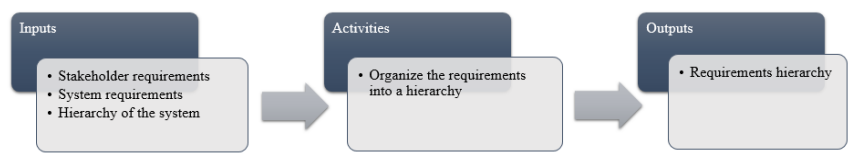

Figure 8: Inputs, activities and outputs of the sixth step

If the system being modified was developed in accordance with a requirements baseline at the development stage, a new baseline shall be created to add the new set of requirements obtained in this step. If a new requirement is equivalent to an existing one and the reported problems show that the system did not meet this requirement, it indicates that the system and/or requirements were not correctly verified and validated, thus an adequate $\mathrm{V} \& \mathrm{~V}$ strategy must be defined. A traceability analysis shall also be performed to identify the links between the new requirements and existing ones. If an existing requirement is affected, the need to modify, revalidate and reverify that requirement must be assessed. 


\subsection{Seventh step of the system modification process}

In this step, a V\&V strategy is defined following the activities shown in fig. 9. Validation of a requirement consists of ensuring that the requirement is justified and relevant to the stakeholders needs. Validating a system means to demonstrate that it meets the requirements, mission or business profile of the stakeholders, as well as their operational scenarios. The stakeholders shall be involved in the validation process. Verifying a requirement is to check the way it is written and the application of its desired characteristics, such as unambiguity, consistency, completeness, uniqueness, traceability, verifiability, among others. Verifying a system is the activity of checking whether its characteristics or properties are in accordance with requirements, architecture and design [1].
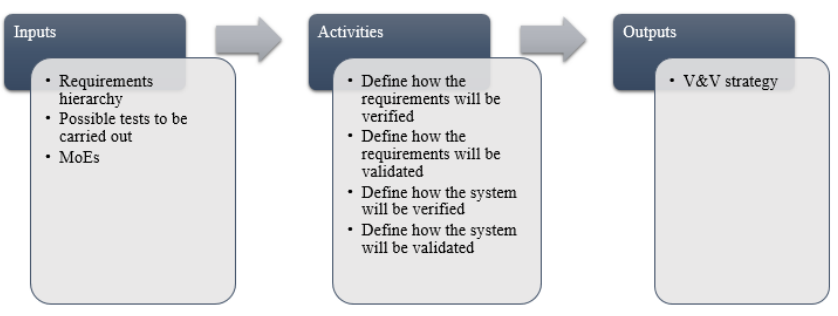

Figure 9: Inputs, activities and outputs of the seventh steps

\section{Case study}

The process of modifying an interior system is different from other systems in terms of measuring customers' perception. Stakeholder concerns about interior systems, e.g. concerns involving appearance and comfort aspects, are often qualitative, which makes measuring the effectiveness of the solution a difficult and time consuming task.

This particularity of interior systems can make the standardization of the system modification process more difficult, and the process becomes dependent on the expertise of its stakeholders. In order to avoid this dependence and to exemplify the approach presented in this paper, a folding table installed on an executive aircraft was chosen to be analysed in the case study. Some customers have reported problems in the folding table of an executive aircraft after the modification of the system through the AS-IS process. The problems were mostly related to damage to the finishing material and to issues involving the functionality of the mechanism.

The current system modification process, i.e. the AS-IS process, used to modify the folding table has been adapted by one of the authors to include the seven steps previously presented in fig. 2. The AS-IS process was modelled according to a methodology that divide process modelling into three phases: data collection, definition of the AS-IS model, and validation of the model. The first phase consists of defining the goal and the detail level of the model, as well as analysing the process. The second phase involves selecting the modelling tools and defining the AS-IS process design. In the third phase, the model is tested and, if needed, some modifications are made [8]. The AS-IS process deficiencies were identified from the information obtained from the interviews with the process stakeholders and from a value analysis of each activity. The deficiencies were prioritize in a gravity, urgency and tendency (GUT) matrix and the activities related to them were replaced by the seven requirements-driven steps. The methodology used herein to model a system modification process integrates process modelling with requirements engineering in the TO-BE process definition. This methodology is shown in fig. 10 .

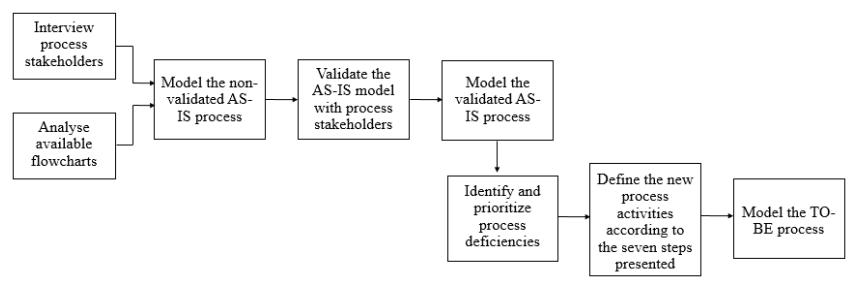

Figure 10: Methodology used to model the AS-IS and TOBE processes

The activities of the TO-BE process have been carried out by one of the authors to simulate the activities to be performed by the engineers if the process were actually implemented. After that, the set of requirements obtained was compared against the reported problems, showing that the TO-BE process addressed several problems that have not been identified through the AS-IS process.

The outcomes of each step for the folding table in operation are presented hereafter. According to the system hierarchy, the folding table can be considered a subsystem of the interior, which in turn is a subsystem of the aircraft, as shown in fig. 11 .

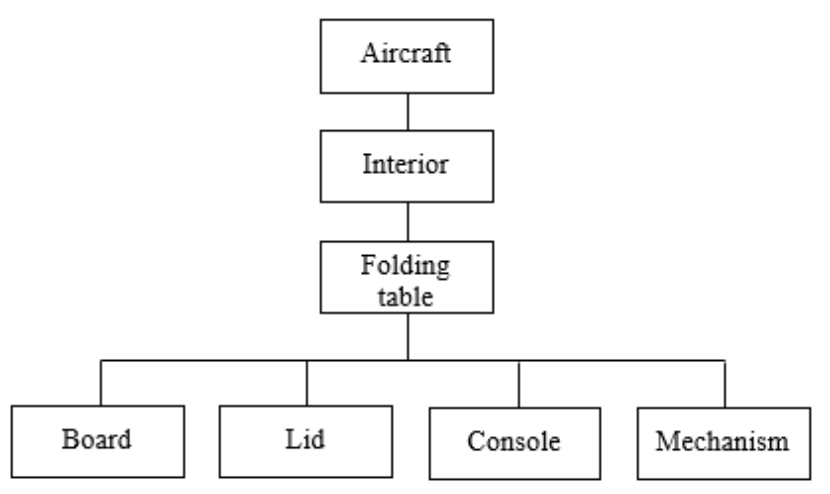

Figure 11: System hierarchy

\subsection{System mission, life cycle and scope of development effort}

In this case study, the mission has been defined in the second level of the hierarchy, because the folding table mission is within the interior mission, which is to provide comfort, practicality, entertainment and safety to passengers from start to finish of the trip. As the life cycle of an aircraft includes all its subsystems, the engineer can use the stages previously presented in fig. 1 . The responsibility for the development of interior systems is shared between the aircraft manufacturer and the interior supplier. Thus, both companies are considered development organizations. The aircraft 
manufacturer also participates solely or partially in the production, certification, training and maintenance stages, characterizing its broad scope of the development effort.

\subsection{Stakeholder analysis}

Based on boilerplates available in the literature [7] [9], the requirement boilerplates shown in Table 1 have been defined and used in writing requirements. The placeholders are the parts that vary from requirement to requirement.

Table 1: Requirement boilerplates for the case study

\begin{tabular}{|c|c|}
\hline Domain & Boilerplate \\
\hline \multirow[t]{5}{*}{ Problem } & $\begin{array}{l}\text { The <stakeholder type> shall be able to } \\
\text { <capability> }\end{array}$ \\
\hline & $\begin{array}{l}\text { While <operational condition >, the } \\
<\text { stakeholder type }>\text { shall be able to } \\
<\text { capability }>\text {... }\end{array}$ \\
\hline & $\begin{array}{l}\text {.. within <performance > of } \\
\text { <event }>\end{array}$ \\
\hline & $\begin{array}{l}\ldots \text { at least <quantity> times per } \\
<\text { unit time> }\end{array}$ \\
\hline & $\begin{array}{l}\ldots \text { for a period of at least }\langle\text { quantity }> \\
\langle\text { unit time }>\end{array}$ \\
\hline
\end{tabular}

Solution The <system > shall (not) <system response>

When <optional precondition> <trigger>, the <system> shall (not) <system response $>$

If <optional preconditions/trigger $\rangle$, then the <system> shall (not) <system response>

While <in an specific state>, the <system> shall (not) <system response>

Where <feature in included $>$, the <system> shall (not) <system response $>$

Figures 12 and 13 show the context diagram of the aircraft interior and the folding table in operation, respectively. The elements of the diagram are the stakeholders of the system in operation and their concerns are listed in bullet points.

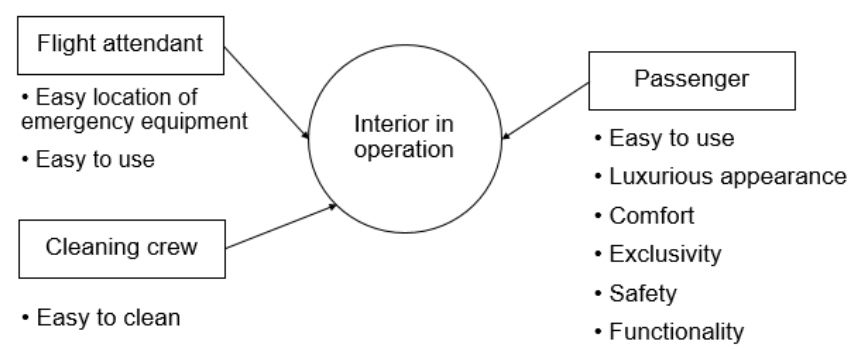

Figure 12: Stakeholders of the aircraft interior in operation and their concerns

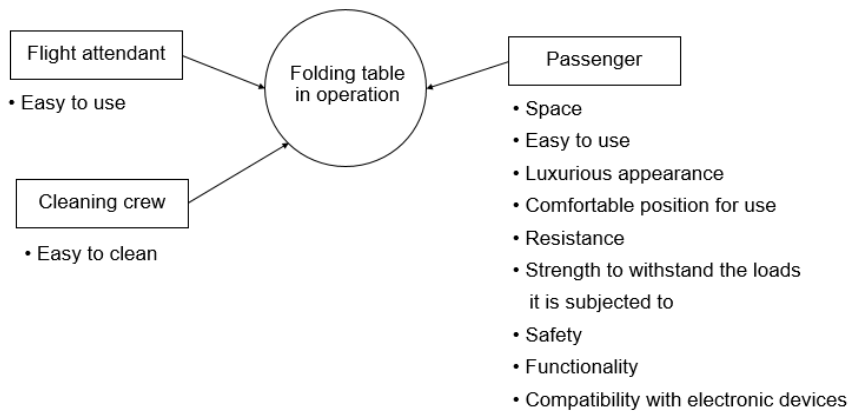

Figure 13: Stakeholders of the folding table in operation and their concerns

Through the diagrams of fig. 12 and 13, two requirements were obtained from the stakeholder passenger and his/her concern about the functionality and luxurious appearance of the system. As these concerns are the same for both the aircraft interior and the folding table, the requirements were stated for the higher level system, so any other interior subsystem should also meet these requirements. Therefore, no differentiation was made between hierarchy levels for stakeholder requirements. The requirements were stated as follows:

- The passenger shall be able to use all aircraft interior features on all flights (type of requirement: capability).

- The passenger shall be able to feel satisfied with the appearance of the aircraft interior (type of requirement: capability).

\subsection{MoEs}

Table 2 shows the MoEs defined from the passenger's concerns about the resistance of the folding table. These MoEs illustrates the particularity of interior systems in quantifying customers' perception. The concept of no permanent damage may vary from customer to customer and the number of cycles can be defined based on flight data and laboratory tests, which must consider the contamination of the finishing material by substances contained in drinks and foods. The normal conditions of use defined in the criteria must be well defined and documented.

Table 2: MoEs for the folding table

\begin{tabular}{ll}
\hline MoE & Criterion \\
\hline $\begin{array}{l}\text { Resistance to } \\
\text { scratching }\end{array}$ & $\begin{array}{l}\text { No permanent scratches on the finishing } \\
\text { material after } X \text { cycles, under normal } \\
\text { conditions of use }\end{array}$ \\
\hline $\begin{array}{l}\text { Resistance to } \\
\text { pressure } \\
\text { marks }\end{array}$ & $\begin{array}{l}\text { No permanent dimples in the finishing } \\
\text { material after } X \text { cycles, under normal } \\
\text { conditions of use }\end{array}$ \\
\hline $\begin{array}{l}\text { Stain } \\
\text { resistance }\end{array}$ & $\begin{array}{l}\text { No permanent stains on the finishing } \\
\text { material after } X \text { cycles under normal } \\
\text { conditions of use }\end{array}$ \\
\hline
\end{tabular}




\begin{tabular}{ll}
\hline Abrasion & No noise indicating abrasion of the \\
resistance & components of the mechanism after $\mathrm{X}$ \\
& cycles, under normal conditions of use
\end{tabular}

\subsection{Functional analysis}

Figures 14 and 15 show the functional context diagram for the interior and folding table in operation, respectively. The analysis has been performed for both levels of hierarchy to properly capture the interface requirements between the folding table and the systems that interact and interfere in its operation. In the diagrams, the direction of the arrows indicates the direction in which ENR and MAT are exchanged between the system under analysis and other systems or stakeholders.

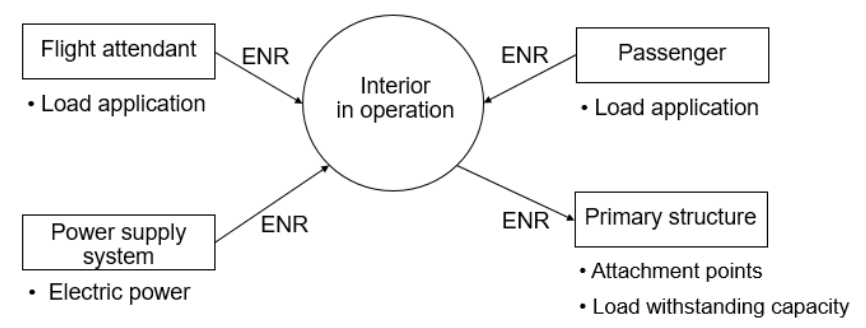

Figure 14: Functional context diagram for the aircraft interior in operation

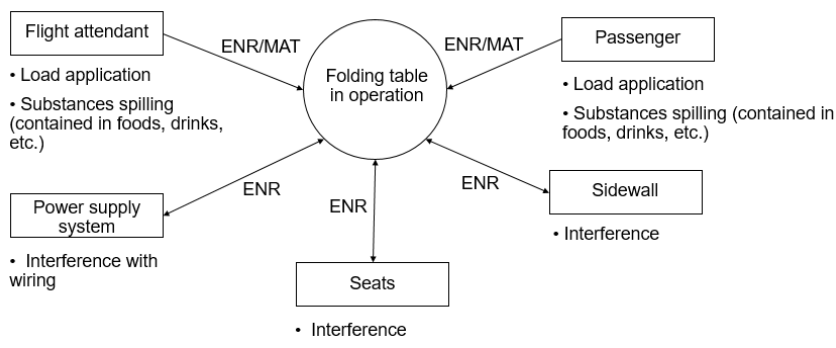

Figure 15: Functional context diagram for the folding table in operation

Table 3 presents the list of events to identify the system functions. The functional structure diagram related to the list of events is shown in fig. 16, whereas the state transition diagram is shown in fig. 17.

Table 3: Event list to identify system functions

\begin{tabular}{ll}
\hline & System in operation \\
\hline System stimulus & System response \\
\hline 1. User press the lid & 1. The system opens the lid \\
\hline $\begin{array}{l}\text { 2. User press the table } \\
\text { board }\end{array}$ & $\begin{array}{l}\text { 2. The system partially lifts } \\
\text { the table board }\end{array}$ \\
\hline $\begin{array}{l}\text { 3. User pull up the table } \\
\text { board }\end{array}$ & $\begin{array}{l}\text { 3. The system locks the } \\
\text { table board when it is } \\
\text { completely released }\end{array}$ \\
\hline $\begin{array}{l}\text { 4. User moves the table } \\
\text { board down }\end{array}$ & $\begin{array}{l}\text { 4. The system locks the } \\
\text { table board horizontally }\end{array}$ \\
\hline
\end{tabular}

5. User unfolds the table
board

5. The system locks the outer part of the table board horizontally

\begin{tabular}{ll}
\hline $\begin{array}{l}\text { 6. User makes use of the } \\
\text { table }\end{array}$ & $\begin{array}{l}\text { 6. The system withstands } \\
\text { user-imposed loads }\end{array}$ \\
\hline $\begin{array}{l}\text { 7. User folds the outer part } \\
\text { of the table board }\end{array}$ & $\begin{array}{l}\text { 7. The system retracts the } \\
\text { outer part over the inner part } \\
\text { of the table board }\end{array}$
\end{tabular}

8. User raises the table 8. The system opens the lid board to the upright position

\begin{tabular}{|c|c|}
\hline $\begin{array}{l}\text { 9. User stows the table } \\
\text { board }\end{array}$ & $\begin{array}{l}\text { 9. The system locks the } \\
\text { table board inside the } \\
\text { console }\end{array}$ \\
\hline 10. User closes the lid & 10. The system is not in use \\
\hline
\end{tabular}

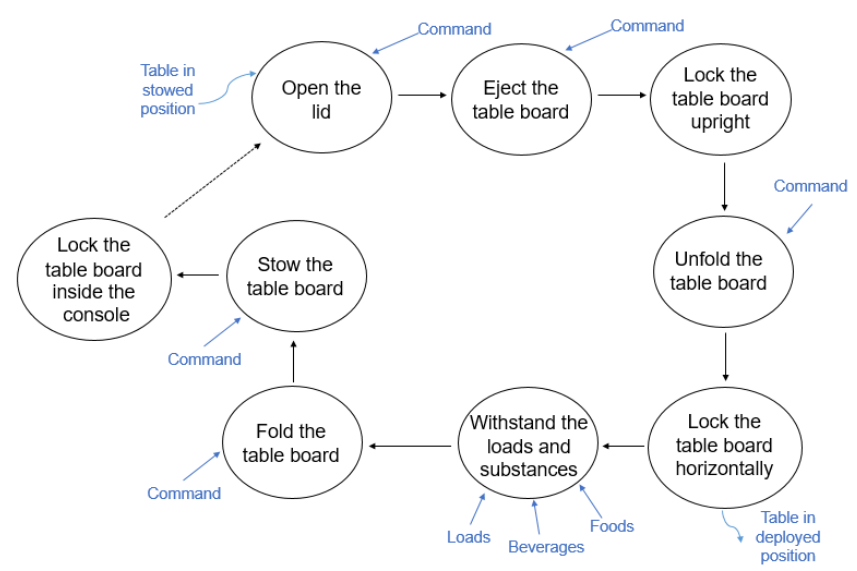

Figure 16: Functional structure diagram of the folding table

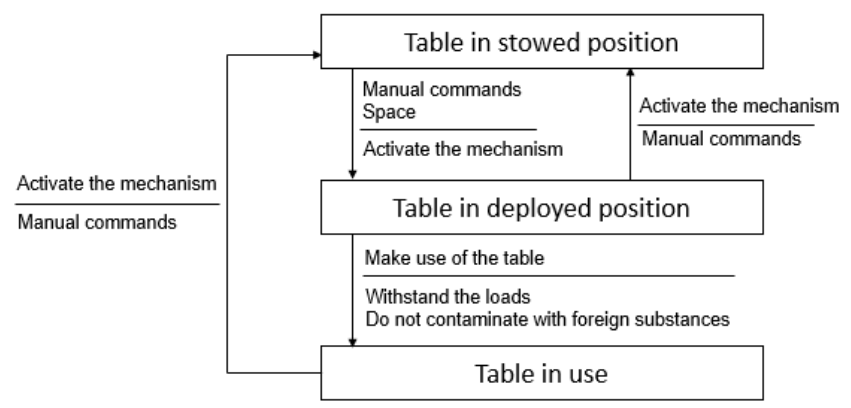

Figure 17: State transition diagram of the folding table

The following requirements have been identified through the functional analysis:

- While under normal conditions of use, the aircraft interior shall operate for $\mathrm{X}$ cycles without damage to the finishing material (type of requirement: functional).

- While under normal conditions of use, the aircraft interior shall operate for X cycles without damaging its functionality (type of requirement: functional). 
- While being stowed by the user, the table shall not have its movement blocked by contact between any parts (type of requirement: interface/constraint).

- When the table board reaches a position of $(\mathrm{X} \pm \mathrm{Y})^{\circ}$, the lid shall be in vertical position $\left(90^{\circ}\right)$ (type of requirement: interface).

- While the table is stowed, the table shall not have contact between parts with finishing material (type of requirement: constraint).

- While the table is stowed, the outer part of the table board shall not have contact with the inner part of the table board (type of requirement: interface/constraint).

- The table shall not have contact between its metal parts and finishing material (type of requirement: interface/constraint).

- While the table is stowed, the table board shall not have contact with the mechanism (type of requirement: interface/constraint).

Regarding the type of requirement, the requirements above were classified as interface requirements because they state the functional relationships that exist among the folding table elements. Requirements whose text contains shall not have also been classified as constraints.

\subsection{Physical analysis}

Figures 18 and 19 show, respectively, the architecture context of the interior and the folding table in operation, in which the physical components responsible for the exchange of ENR and/or MAT were identified.

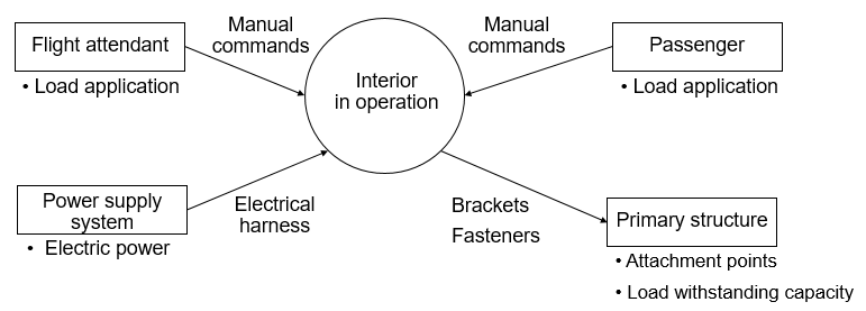

Figure 18: Architecture context for the aircraft interior in operation

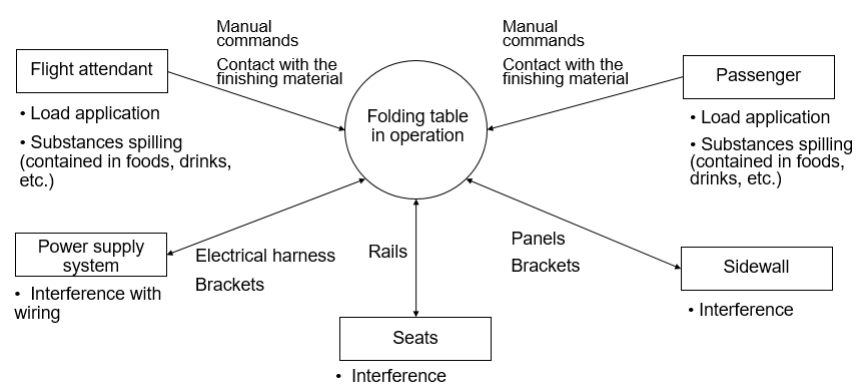

Figure 19: Architecture context for the folding table in operation

The following requirements have been identified through the physical analysis.
- The table shall absorb the impacts coming from contact with the sidewall panel (type of requirement: interface).

- The table shall not have abrasion damage after X cycles (type of requirement: constraint).

These requirements were classified as interface or constraint requirements, similar to the classification used in the functional analysis. The difference here is that the interface requirement states a physical relationship between the folding table and the sidewall, which is an environment element.

\subsection{Requirements hierarchy}

The stakeholder requirements and the set of system requirements were organized into a hierarchy, as shown in fig. 20. An identification number was assigned to each requirement, in which the first digit corresponds to the hierarchy level as follows: 1) first level - stakeholder requirements; 2) second level - interior requirements; 3 ) third level - folding table requirements; 4) fourth - table elements requirements. The second digit is used to differentiate the requirements within the same level.

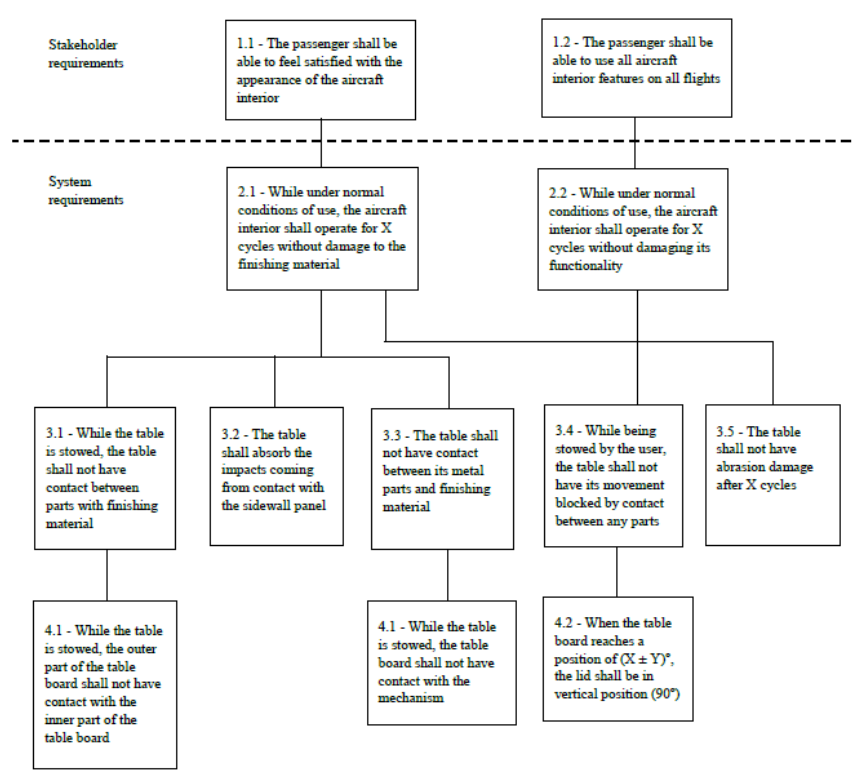

Figure 20: Requirements hierarchy

The requirements hierarchy focus on finishing materials and mechanism interfaces issues to solve the problems reported from customers. However, many other requirements could be identified through the stakeholders, functional and physical analyses. Thus, for each case, the engineers will guide the direction of the analyses with the aim of addressing the reported problems. If some reported problem is related to any organizational issue, all analyses performed for the system must also be carried out for the organizations.

In case the aircraft subsystem is developed and manufactured by a supplier, both the aircraft manufacturer and the supplier will be involved in the system modification process. In this case study, for instance, the folding table supplier should 
break down the folding table requirements into lower-level requirements until the level of components.

\subsection{V\&V strategy}

The system and the requirements could be verified and validated as follows:

Requirements verification: the requirements have the characteristics of a good requirement (e.g. unique, traceable, unambiguous, etc. [5]) and have been written using the boilerplates of Table 1 .

Requirements validation: the requirements are relevant to the needs of the stakeholders in the hierarchy shown in fig. 20, in which all system requirements are linked to stakeholder requirements.

System verification: this process must begin at the lowest level of the hierarchy and consists of verifying that the system meets the system requirements. The requirements could be verified through analyses, tests, simulations, traceability, among other methods.

System validation: the system meets the stakeholder requirements by complying with their children requirements, as well as by achieving the effectiveness expected by the customer, defined through the MoEs.

\subsection{Outcome}

A set of requirements was obtained after executing the activities of the proposed system modification process. The reported system problems were compared against these requirements. In case the problem would be avoided by meeting one or more requirements, it was considered that the problem would be solved. The outcome shows that the new process would potentially avoid $60 \%$ of the problems. It is believed that this figure would be even greater if the process were actually implemented. This is because the stakeholder, functional and physical analyses would not be carried out by only one person, but rather by a multidisciplinary team with the support of all process stakeholders to obtain a sufficient set of requirements.

\section{Conclusion}

The approach presented in this paper can be used by an organization to define or improve its system modification process in order to obtain a solution of high effectiveness. By achieving the effectiveness desired by customers, additional costs of a second rework are avoided and customer satisfaction is increased. The steps of the system modification process are comprehensive and can be tailored to fit different types of systems and organizations.

In addition to contributing to the industry by presenting a system modification process, this paper also contributes to the literature by presenting the use of requirements engineering to modify systems at an advanced life cycle stage. Instead of covering the whole life cycle, this work showed the importance of defining new requirements to modify an aircraft subsystem at the operation stage.

The steps of the system modification process proposed herein proved viable with the case study outcome, which showed a potential reduction of the problems reported during the system operation.

\section{References}

[1] INCOSE, Systems engineering handbook: a guide for system life cycle processes and activities, 4th red., San Diego: John Wiley \& Sons, 2015.

[2] S. Dekker, Drift into failure: from hunting broken components to understanding complex systems, Boca Raton: Taylor \& Francis, 2011.

[3] M. M. N. Zenun, Modelo para obtenção do conjunto suficiente de requisitos no desenvolvimento de sistemas de aeronaves, São José dos Campos, SP: Instituto Tecnológico de Aeronáutica (ITA), 2015.

[4] G. Loureiro, A systems engineering and concurrent engineering framework for the integrated development of complex products, Loughborough: Loughborough University, 1999, p. 362.

[5] A. T. Bahill och A. M. Madni, Tradeoff Decisions in System Design, Los Angeles, CA: Springer, 2017.

[6] G. Loureiro, A. G. Adinolfi, C. E. V. Ribeiro och R. C. B. d. Andrade, "System concurrent engineering for the development of an aeronautical navigation system," Product: Management \& Development, vol. 8, nr 2, pp. 107-122, 2010.

[7] E. Hull, K. Jackson och J. Dick, Requirements engineering, London: Springer, 2011.

[8] C. S. T. Amarala, H. Rozenfeldb, J. M. H. Costab, M. d. F. d. A. Magon och Y. M. Mascarenhas, 'Improvement of radiology services based on the process management approach," European Journal of Radiology, nr 78, p. 377-383, 2011.

[9] J. Dick och J. Llorens, ”Using statement-level templates to improve the quality of requirements," i 24th International Conference on Software \& Systems Engineering and their Applications, Paris, 2012. 